\title{
Editorial, special issue of NETYS 2015
}

\author{
Ahmed Bouajjani ${ }^{1} \cdot$ Hugues Fauconnier $^{1}$
}

Published online: 24 July 2019

๑) Springer-Verlag GmbH Austria, part of Springer Nature 2019

The aim of the International Conference on Networked Systems (NETYS) is to bring together researchers and engineers from both the theory and practice of distributed and networked systems. The scope of the conference covers all aspects related to the design and the development of these systems, including multi-core architectures, concurrent and distributed algorithms, parallel/concurrent/distributed programming, distributed databases, big data applications and systems, cloud systems, networks, security, and formal verification.

This volume contains full versions of a selection of best papers presented at the third International Conference on Networked Systems, NETYS 2015, which took place in Agadir, Morocco, in May 13-15, 2015. The articles presented in this special issue of NETYS 2015 address some of the most important issues in networked systems.

The article "Linearizability is EXPSPACE-complete" by Jad Hamza addresses the problem of verifying concurrent objects, and more precisely the fact that all executions of a given implementation of such an object are linearizable. Linearizability is a fundamental correctness criterion for concurrent systems that was introduced in an article by Herlihy and Wing in 1990. In 1996, Alur et al. proved that verifying linearizability for finite-state implementations of concurrent objects is in EXPSPACE. Jad Hamza establishes in the paper presented in this volume that the problem is actually EXPSPACE-hard, closing a long standing open question.

The article "Optimal Torus Exploration by Oblivious Robots" by Stéphane Devismes, Anissa Lamani, Franck Petit and Sebastien Tixeuil addresses the possibility of having terminating algorithms for networks exploration by a team of mobile robots. The authors investigate this question for torus-shaped networks (a torus being a special case of a two dimensional ring), and considered robots that are weak in the sens that they are anonymous, uniform and oblivious. The authors first show impossibility results stating mainly that it is impossible to explore any torus of arbitrary size with less than four robots. Then, they provide a generic probabilistic algorithm that solves the problem with four robots in any torus of size at least $7 \times 7$.

\footnotetext{
Ahmed Bouajjani

abou@irif.fr

1 Université Paris Diderot, Paris, France
} 
The article "EPiC: Efficient Privacy-Preserving Counting for MapReduce" addresses the issue of protecting outsourced data in the cloud. The authors present EPiC, a practical protocol for the privacy-preserving evaluation of a fundamental operation on data sets: frequency counting. EPiC's privacy is formally proven and a significant experimental evaluation has been carried out, showing only a low overhead compared to non-private counting, which attests to EPiC's efficiency.

The article "A Fully Distributed Learning Algorithm for Power Allocation in Heterogeneous Networks" proposes an algorithm for optimizing power allocation in heterogeneous networks. The problem is formalized in a game-theoretic framework, and an heuristic-based distributed algorithm is proposed to solve efficiently the search for an optimal solution. An experimental evaluation of the algorithm is carried out showing its qualitative and quantitative properties.

Preliminary versions of the four articles of this Special Issue appeared in the proceedings of the NETYS 2015 conference that were published as the volume No. 9466 of the Lecture Notes in Computer Science series, Springer 2015, ISBN 978-3-31926849-1.

We would like to thank the authors of the articles in this volume for their valuable contributions, as well as the reviewers of this special issue for their thorough and rigorous work.

Publisher's Note Springer Nature remains neutral with regard to jurisdictional claims in published maps and institutional affiliations. 